\title{
Obstáculos para la práctica de la medicina basada en la evidencia
}

Obstacles to answering doctors questions about patient care with evidence: qualitative study. Ely J, Osheroff J, EbellM. BMJ;2002;324: 710-713.

\section{Objetivo}

Describir los obstáculos con que se encuentran los médicos cuando intentan responder con evidencia preguntas clínicas reales de su práctica cotidiana.

Diseño

Investigación cualitativa.

Lugar

Centros de atención primaria de lowa, EEUU.

\section{Participantes}

Nueve médicos generalistas, 14 médicos de familia y dos bibliotecarios.

Sujetos

Ciento tres médicos de familia

\section{Métodos}

Inmediatamente luego de la entrevista un observador inquiría a los médicos qué preguntas clínicas habían surgido en esa consulta. De las 1101 preguntas recolectadas, algunas extremadamente precisas (ej. ¿cuál es la dosis de cierto medicamento?) y otras muy vagas (ej. ¿qué será esta erupción de este paciente?) fueron seleccionadas 200 en forma aleatorizada. A través de un proceso iterativo de revisión y clasificación se desarrolló un método para la identificación de las que fueran potencialmente contestables con evidencia.

Para responder las preguntas clínicas cada investigador sólo consultó libros de texto, recursos informatizados, revistas médicas, etc. y sin hacer consultas a colegas trabajó en forma independiente. Cada búsqueda fue considerada completa cuando cada participante estimó que no había pasado por alto evidencia relevante sobre la pregunta.Mientras lo hacían, iban anotando los obstáculos que se les interponían en el camino. A través de las notas de los investigadores, las frustraciones que luego reportaron y las dificultades que figuraban en la bibliografía fueron descriptos y organizados los obstáculos a través de la técnica de análisis cualitativo de textos. Para validar los resultados se pidió a dos médicos y a dos bibliotecarios más que respondieran cuatro preguntas adicionales de la selección original; se preguntó a 21 médicos que ejercían la práctica clínica que describieran por escrito los problemas que encontraban al intentar responder las preguntas que a ellos les surgían y se observó durante cuatro períodos de medio día de su práctica a cada uno de cuatro médicos a quienes se había solicitado que pensaran en voz alta mientras atendían a sus pacientes.

\section{Resultados}

De las 200 preguntas que habían sido seleccionadas en forma aleatorio, $106(53 \%)$ fueron identificadas como pasibles de ser respondidas con evidencia. Hubo acuerdo en tres criterios que debían cumplirse para que esto fuera posible y para que valiera la pena hacerlo: 1) clara formulación de la pregunta;

2) alta probabilidad de encontrar evidencia de buena calidad para responderla;

3) potencial impacto en los cuidados del paciente.

Los obstáculos fueron clasificados en las cinco categorías que se describen en el cuadro 1.

Cuadro 1:obstáculos para la práctica de la medicina basada en la evidencia (MBE).

1) Reconocimiento de la necesidad de información: no se da cuenta lo que no sabe o se da cuenta y lo niega por incomodidad, falta de tiempo o vergüenza.

2) Formulación de la pregunta: dificultad para seleccionar preguntas que puedan ser respondidas a través de los recursos de la MBE* y para formularlas a través del formato PICR (paciente, intervención (o exposición), comparación, resultado).

3) Búsqueda de información: la búsqueda de información consume más tiempo que la interconsulta con un colega, existe incertidumbre sobre donde buscar información, hay dificultades para interaccionar con los recursos bibliográficos y algunas preguntas que surgen en el consultorio no han sido formuladas por los estudios disponibles.

4) Formulación de la respuesta: la información encontrada no es la más adecuada (ej. no está dirigida a médicos) 0 existen dificultades para sintetizarla y poder concluir una respuesta.

5) Aplicación al cuidado del paciente: falta de confianza del médico sobre la información que ha hallado.

*Son preguntas imposibles de ser respondidas a través de los recursos de la MBE las que son extremadamente inespecíficas (ej.¿qué será esta erupción? ¿qué le pasa a este paciente?).

\section{Conclusiones}

Se encuentran muchos obstáculos al preguntarse y responderse preguntas clínicas acerca del cuidado de los pacientes.Tomar en cuenta estos obstáculos podría llevar a un mejor cuidado del paciente a través del mejoramiento de los recursos de información con orientación clínica.

Fuente de financiamiento: American Academy of Family Physicians y National Library of Medicine.

\section{Comentario}

Como estrategia de aprendizaje y educación médica continua, la medicina basada en la evidencia intenta obtener en el menor tiempo y con el menor esfuerzo posible respuestas confiables a preguntas específicas 1 . Como mostró esta investigación cualitativa, una de cada dos preguntas que surgen durante la práctica clínica podría ser potencialmente respondida a través de MBE.Sin embargo, durante nuestra práctica intentamos responder nuestras dudas principalmente haciéndoselas a otros colegas a través de una pregunta "de pasillo" o en forma de una interconsulta formal o bien, buscando la respuesta en algún libro de texto. Este trabajo mostró que suele haber dificultades para convertir una pregunta clínica referida a un individuo particular (ej.qué test diagnóstico le debo solicitar a "este paciente" o qué medicamento le debo indicar a "este paciente") a una pregunta referida a una población de pacientes parecidos al míoz.El no cumplir satisfactoriamente este proceso dificulta el hallazgo de una respuesta con las herramientas disponibles ya que la información existente suele ser poblacional y no individual.

En cuanto a la falta de tiempo, un obstáculo que considero de los más importantes a causa del vértigo con el que nos vemos obligados a atender a nuestros pacientes, suele resultar más rápido y práctico realizar una interconsulta que llevar a cabo los distintos pasos que conducen a hallar la respuesta respaldada por la mejor evidencia disponible (alternativas en realidad no excluyentes sino complementarias). Si a esto le sumamos que no nos resulta demasiado atractivo hacernos cargo de problemas de salud que no manejamos con soltura, esta sensación ayuda a disparar el pedido de la interconsulta a otro colega con mayor experiencia en el tema.A su vez, la repetición de este proceso consolida nuestra falta de habilidad para el manejo de este tipo de problema que terminamos prefiriendo derivar antes que aprender a manejar.

Volvemos a insistir en que la medicina basada en la evidencia nunca puede reemplazar a la experiencia y a la destreza clínica, pero sí debe complementar estas habilidades. Dicho de otro modo, si no se sabe interrogar y revisar al paciente, si no se tiene la experiencia para poder clasificarlo en las categorías clínicas que permiten pronosticar como evolucionará sin realizarle intervención alguna o administrándole algún tratamiento o bien qué probabilidad existe de que quede mejor categorizado luego de realizarle tal o cual prueba diagnóstica, no habrá pregunta clínica lo suficientemente bien construida ni búsqueda bibliográfica que pueda dar una respuesta útil.Por otro lado, si carecemos de la capacidad para evaluar objetivamente la evidencia disponible para avalar una decisión o una recomendación, corremos el peligro de caer en prácticas predominantemente sesgadas por experiencias personales.

Dr. Sergio Terrasa [ Unidad de Medicina Familiar y Preventiva del Hospital Italiano de Buenos Aires ]

Referencias

1.Evidence-Based Medicine Working Group.Evidence-based medicine:a new approach to teaching the practice of medicine-JAMA.1992:268:2420-5.

2. Richardson WS, Wilson MC, Nishikawa J.The well built clinical question: a key to evidence-based decisions.ACP Journal Club. 1995;123:A12. 